\title{
Cloning and differential expression analyses of Cdc42 from sheep
}

\author{
Yong-Jie Yang ${ }^{1,2}$, Zeng-Shan Liu ${ }^{1}$, Shi-Ying Lu ${ }^{1}$, \\ Pan $\mathrm{Hu}^{1}$, Chuang $\mathrm{Li}^{2}$, Waqas Ahmad ${ }^{1,5}$, Yan-Song $\mathrm{Li}^{1}$, \\ Yun-Ming $\mathrm{Xu}^{1,3}$, Feng Tang ${ }^{1,4}$, Yu Zhou ${ }^{1}$, Hong-Lin Ren ${ }^{1}$
${ }^{1}$ Key Laboratory of Zoonosis Research, Ministry of Education, Institute of Zoonosis, College of Veterinary Medicine, Jilin University, Changchun 130062, China
${ }^{2}$ Department of Food Science, College of Agriculture, Yanbian University, Yanji 133002, China
${ }^{3}$ Jiangsu Polytechnic College of Agriculture and Forestry, Jurong 212400, China
${ }^{4}$ College of Animal Husbandry and Veterinary Medicine, Liaoning Medical University, Jinzhou 121001, China
${ }^{5}$ Section of Epidemiology and Public Health, College of Veterinary and Animal Sciences, Jhang 35200, Pakistan \\ renhl@jlu.edu.cn
}

Received: September 7, 2017 Accepted: February 14, 2018

\begin{abstract}
Introduction: Serological diagnosis of brucellosis is still a great challenge due to the infeasibility of discriminating infected animals from vaccinated ones, so it is necessary to search for diagnostic biomarkers for differential diagnosis of brucellosis. Material and Methods: Cell division cycle $42(\mathrm{Cdc} 42)$ from sheep (Ovis aries) (OaCdc42) was cloned by rapid amplification of cDNA ends (RACE), and then tissue distribution and differential expression levels of OaCdc42 mRNA between infected and vaccinated sheep were analysed by RT-qPCR. Results: The full-length cDNA of OaCdc42 was 1,609 bp containing an open reading frame (ORF) of $576 \mathrm{bp}$. OaCdc42 mRNAs were detected in the heart, liver, spleen, lung, kidneys, rumen, small intestine, skeletal muscles, and buffy coat, and the highest expression was detected in the small intestine. Compared to the control, the levels of OaCdc42 mRNA from sheep infected with Brucella melitensis or sheep vaccinated with Brucella suis $\mathrm{S} 2$ was significantly different $(\mathrm{P}<0.01)$ after 40 and 30 days post-inoculation, respectively. However, the expression of OaCdc42 mRNA was significantly different between vaccinated and infected sheep $(\mathrm{P}<0.05$ or $\mathrm{P}<0.01)$ on days: 14,30 , and 60 post-inoculation, whereas no significant difference $(\mathrm{P}>0.05)$ was noted 40 days post-inoculation. Moreover, the expression of OaCdc42 from both infected and vaccinated sheep showed irregularity. Conclusion: $\mathrm{OaCdc} 42$ is not a good potential diagnostic biomarker for differential diagnosis of brucellosis in sheep.
\end{abstract}

Keywords: sheep, brucellosis, Cdc42, cloning, differential expression.

\section{Introduction}

Brucellosis caused by Brucella spp. is a worldwide zoonosis, which affects livestock, wildlife, and humans in great numbers (3). As a global epidemic, brucellosis has been reported in over 170 countries (18). Despite being endemic in many countries, the disease remains under-reported or overlooked because of underdiagnosis (5), therefore leading to important economic losses and posing a severe health threat. At present, vaccination is one of the most effective measures to reduce the prevalence of brucellosis (3). However, vaccine strain can induce antibodies that interfere with the serological tests leading to failure in discriminating vaccinated animals from naturally infected ones $(3,18)$. Thus, it is necessary to search for diagnostic biomarkers allowing to distinguish between vaccinated and infected animals.

Cdc42 is a small GTPase, a member of the Rho subfamily, which can act as a molecular switch to control signal transduction pathways by cycling between an inactive state (GDP-bound) and an active state (GTPbound) (10). In GTP-binding form, Cdc42 interacts with its downstream effectors to induce diverse cellular biological actions, including lamellipodia, filopodia and stress fibre formation (20), cell polarity (4), actin polymerisation (17), transport and endocytosis (19). As a key regulator of cellular actin dynamics, Cdc42 plays an important role in host defence $(10,20)$. Pathogen 
invasion is an actin-dependent process; $\mathrm{Cdc} 42$ can trigger extensive rearrangements of actin cytoskeleton to prevent bacteria or viruses from invading host cells (10, $16,17)$. Many viruses, such as human immunodeficiency virus type 1 (HIV-1), respiratory syncytial virus (RSV), and Ebola virus (EBOV), have evolved to hijack Cdc42 to invade host cells (17). Listeria monocytogenes can induce downregulation of host $\mathrm{Cdc} 42$ to promote Listeria cell-cell spread (16). Furthermore, Cdc42 regulates key signalling pathways by interacting with various effector proteins, such as NF- $\mathrm{kB}$, NOD1, c-Jun N-terminal kinase (JNK), and mitogen-activated protein kinase (MAPK) pathways (9). These pathways tightly control cell proliferation, migration, differentiation, and morphogenesis. Cdc42 was reported to be overexpressed in several different cancers that contributed to tumourigenesis and cancer progression (21). Even though $\mathrm{Cdc} 42$ is closely associated with infectious diseases and cancers; there are no reports about the relationship between $\mathrm{Cdc} 42$ and brucellosis until now. In our previous study, a subtractive cDNA library of buffy coat from Brucella-infected and vaccinated sheep was constructed by suppression subtractive hybridisation (SSH), and a partial cDNA of $\mathrm{Cdc} 42$ gene was screened and sequenced.

In this study, we cloned $\mathrm{Cdc} 42$ from sheep $(\mathrm{OaCdc} 42)$ by rapid amplification of cDNA ends (RACE), and then analysed tissue distribution and differential expression levels of OaCdc42 mRNA by RT-qPCR between infected sheep challenged with $B$. melitensis and sheep vaccinated with $B$. suis $\mathrm{S} 2$. Our aims were to discover potential diagnostic biomarkers to discriminate vaccinated sheep from those infected with virulent Brucella.

\section{Material and Methods}

Bacteria. B. melitensis (smooth virulent strain, Bm) was isolated from naturally infected sheep. B. suis S2 (live rough avirulent strain, S2) was purchased from the Harbin Pharmaceutical Group (China). The preparation of bacteria was described by Yang et al. (22).

Animals. A total of nine male sheep (Ovis aries), healthy and negative for Bm and S2, were used. They were randomly divided into three groups, including infected group challenged with $\mathrm{Bm}$, vaccinated group inoculated with S2, and control group injected with sterile PBS. The dose and manner of injection were applied according to Yang et al. (22). Each group was raised separately under the same breeding conditions.

Samples collection. Heparinised peripheral blood and tissue samples (heart, liver, spleen, lung, kidneys, rumen, small intestine, skeletal muscles) from healthy sheep were collected according to Yang et al. (22). All samples were stored at $-80^{\circ} \mathrm{C}$ until use.

Cloning of OaCdc42 cDNA. According to fractional cDNA of $\mathrm{OaCdc} 42$ from the SSH cDNA library (22), its full-length cDNA was amplified by RACE using the SMARTer ${ }^{\text {TM }}$ RACE cDNA Amplification Kit (Clontech, USA) following the manufacturer's instructions. The primers for the RACE were listed in the Table 1.

Sequence analysis. The full-length of $\mathrm{OaCdc} 42$ cDNA and its predicted protein sequences were analysed using DNAStar5.0 software (DNASTAR, USA). The BLASTx algorithm was used to search for the homology of nucleotide and protein sequences of $\mathrm{OaCdc} 42$ (http:/www.ncbi.nlm.nih.gov/BLAST/). NCBI's CDSearch service was used to analyse the conserved domains (CDs) of $\mathrm{OaCdc} 42$ (http://www.ncbi.nlm.nih. gov/Structure/cdd/cdd.shtml). Multiple alignments were analysed by ClustalX 2.0 software (UCD, Ireland). A phylogenetic tree was constructed using MAGA5.1 software (Sudhir Kumar, USA). The signal peptides, transmembrane domains, phosphorylated sites, and higher structure were predicted according to bioinformatics referred by Yang et al. (22).

Reverse transcription quantitative real-time PCR (RT-qPCR). RT-qPCR was used to investigate tissue distribution and differential expression of OaCdc42 mRNA. Two pairs of primers used for qPCR were also listed in Table 1 . OaCdc42 cDNA was amplified to obtain $233 \mathrm{bp}$. $\beta$-actin cDNA from sheep (GenBank accession number U39357), used as an endogenous control, was amplified to obtain $337 \mathrm{bp}$. Reaction conditions and reaction systems, as well as calculation of relative gene expression were described by Yang et al. (22). All tests were run in triplicate.

Table 1. Primers used for PCR amplification

\begin{tabular}{lll}
\hline Primer & Nucleotide sequence (5'-3') & Method \\
\hline Cdc42 F1 & GTGTGAGACAAGGCCCGTAGGTATG & 3'- RACE; outer \\
Cdc42 F2 & TGGCCCCTTCCCCTCTCAATACTAG & 3'-RACE; inner \\
Cdc42 R1 & GCCATACCTACGGGCCTTGTCTCAC & 5'-RACE; outer \\
Cdc42 R2 & AGGTGCAGGGCATTTGTCATTATTG & 5'-RACE; inner \\
Cdc42 F' & GTTGTTGTGGGTGATGGTGCTGTTG & Real-time PCR \\
Cdc42 R' & CACTGAGAGGCAGACCAGAAACACG & Real-time PCR \\
$\beta$-actin F' & CCCAAGGCCAACCGTGAGAAGATGA & Real-time PCR \\
$\beta$-actin R' & CGAAGTCCAGGGCCACGTAGCAGAG & Real-time PCR \\
\hline
\end{tabular}


$\mathbf{A}$

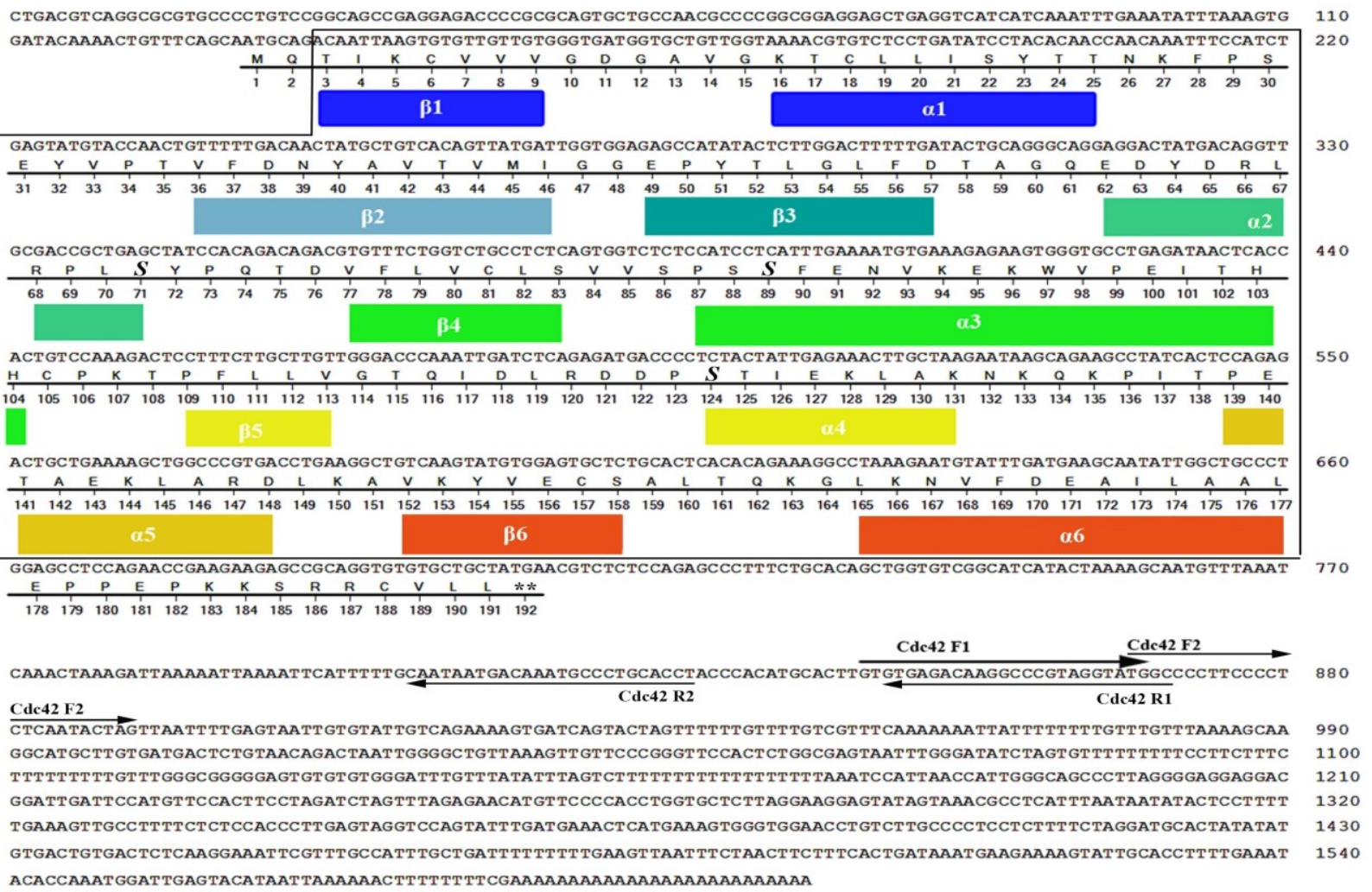

B

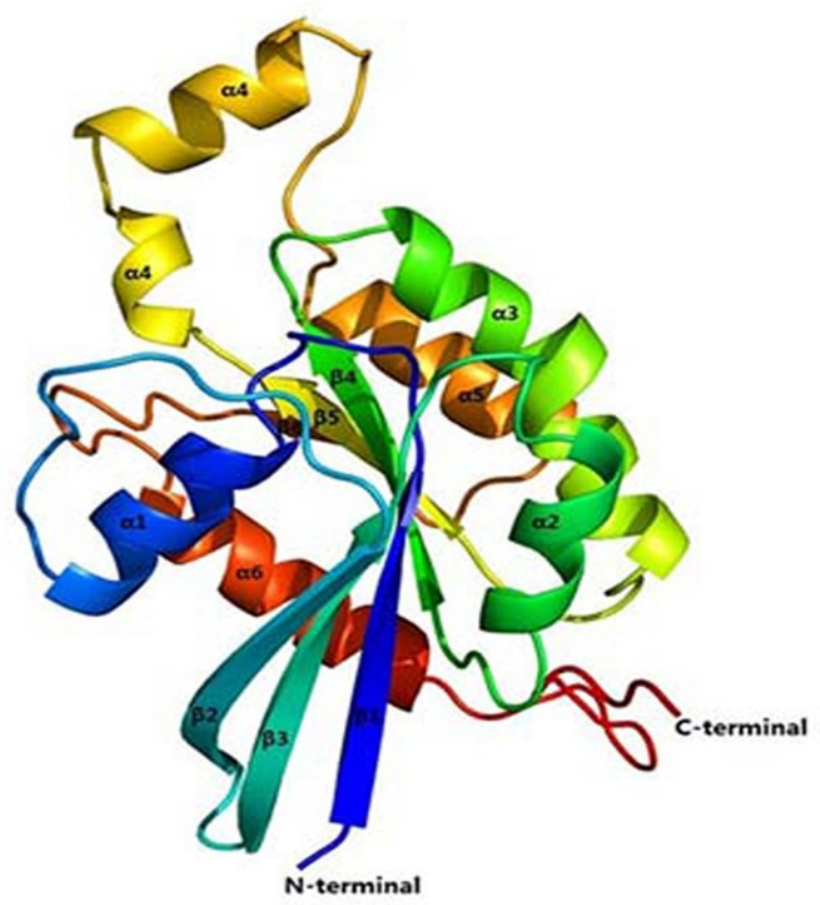

Fig. 1. Sequence analyses of OaCdc42. A. The full-length cDNA and deduced amino acid sequences of OaCdc42. GenBank accession number for OaCdc42 cDNA was KC425615. The conserved domain (CD) was in the frame. Double asterisks $\left({ }^{* *}\right)$ represent stop codon (TGA). Primers for 3'-, 5'-RACE are marked with underlined arrows. " $S$ " in bold and italics indicates the predicted phosphorylated sites. Residue numbers of secondary structure are coloured per domain as in panel. B. The three-dimensional structure of OaCdc42. Each of $\alpha$ - helices and $\beta$-sheet is indicated by a different colour

Statistical analysis. SPSS software version 13.0 (SPSS, USA) was used to analyse data and Student's $t$ test was used to calculate $\mathrm{P}$ values. Differences between individual group means were analysed by a Repeated Measures ANOVA. The differences were considered statistically significant at $\mathrm{P}<0.05$ or $\mathrm{P}<0.01$. Data were presented as mean $\pm \mathrm{SD}$. 


\section{Results}

Sequence characterisation of OaCdc42 cDNA. The full-length of $\mathrm{OaCdc} 42$ cDNA was the first identified and registered in GenBank with the accession number KC425615. Its full-length was $1,609 \mathrm{bp}$ containing $130 \mathrm{bp}$ of 5'-untranslated region (UTR), $576 \mathrm{bp}$ of ORF, and 903 bp of 3'-UTR. The ORF encoded a putative protein of 191 amino acid residuals with a deduced molecular weight (MW) of $21.22 \mathrm{kDa}$ and theoretical isoelectric point $(\mathrm{pI})$ of 6.38 . OaCdc42 was predicted to contain a conserved domain from $\mathrm{Thr}^{3}$ to $\mathrm{Leu}^{177}$ (Fig. 1A), including GTP/ $\mathrm{Mg}^{2+}$ binding site, GTPase-activating protein (GAP) interaction site, guanine nucleotide exchange factor (GEF) interaction site, Par6 cell polarity protein interaction site, Cdc42/Rac-interactive binding (CRIB) effector interaction site, ACK tyrosine kinase interaction site, guanine nucleotide dissociation inhibitor (GDI) interaction site, Switch I and II region, and G1, 2, 3, 4, and 5 boxes. Three serine phosphorylated sites were predicted to locate in $\mathrm{Ser}^{71}, \mathrm{Ser}^{89}$, and $\mathrm{Ser}^{124}$ (Fig. 1A). $\mathrm{OaCdc} 42$ was predicted to be a spherical protein composed of six $\alpha$ - helixes and six $\beta$ - strands (Fig. 1B). In addition, there was no transmembrane domain and signal peptide to $\mathrm{OaCdc} 42$, which indicated that OaCdc42 might not be a secreted or membrane protein.

Multiple alignments revealed that the deduced amino acid sequence of $\mathrm{OaCdc} 42$ protein showed a high homology with other known $\mathrm{Cdc} 42$, and the highest identity of $99 \%$ was with Homo sapiens (NP_001782) (Fig. 2). A phylogenetic tree was constructed using the neighbour-joining (NJ) method based on multiple alignments. The result revealed that there were close genetic relations of $\mathrm{OaCdc} 42$ with other mammals, especially with Homo sapiens, which was in agreement with the result of multiple alignments (Fig. 3).
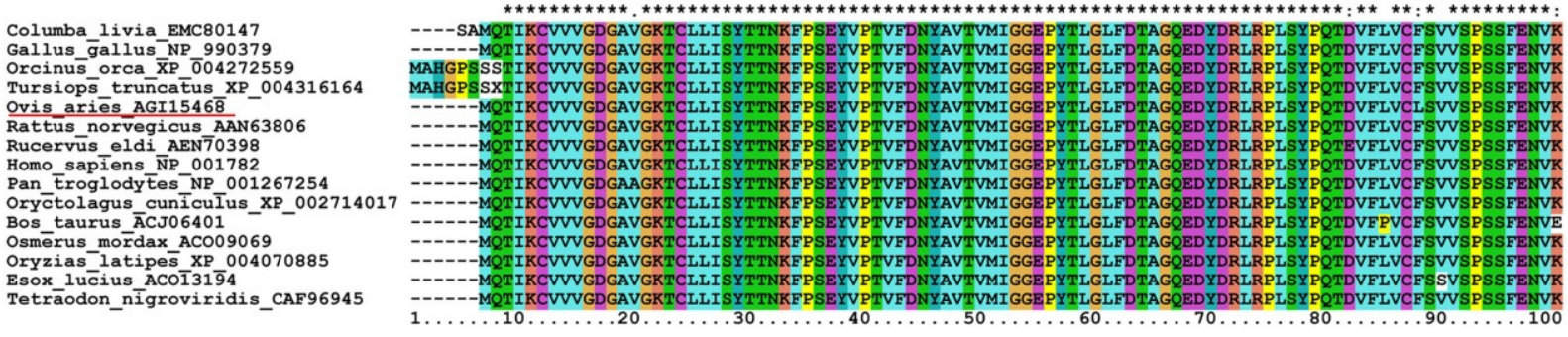

Columba_livia_EMC80147 Gallus_gallus NP 990379 Tursiop_orca X X Ovis aries_AGI15468 Rattüs norvegicus AAN6380
Rucervus eldi AEN7 70398 Homo_sapiens_NP 001782 Pan_troglodytes_NP_001267254 Oryc̄tolagus cuñīcuĨus_XP_002714017 Osmerus mor Oryzias_latipes XP_004070885 Esox lucius ACŌ̄ $31 \overline{9}$ Tetrāodon_nígroviridis_CAF96945

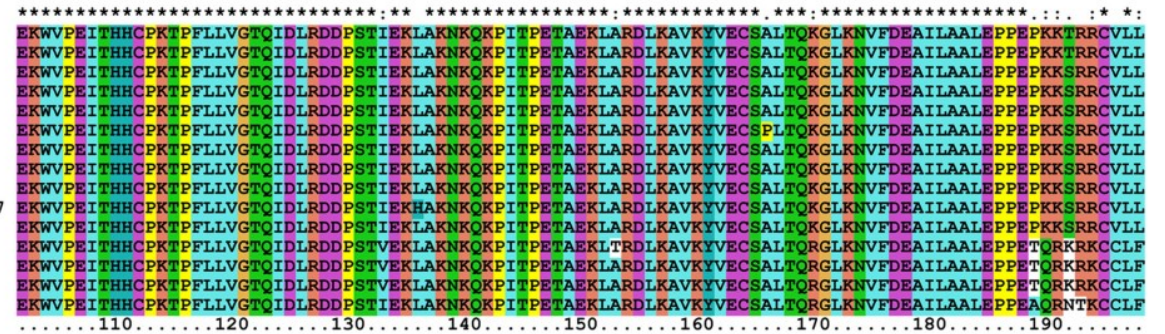

Fig. 2. Multiple alignments of Cdc42 between sheep and other species. The amino acid sequence of OaCdc 42 is underlined. $(*)-100 \%$ identical; $(:)$ - highly conserved; (.) - semi-conserved
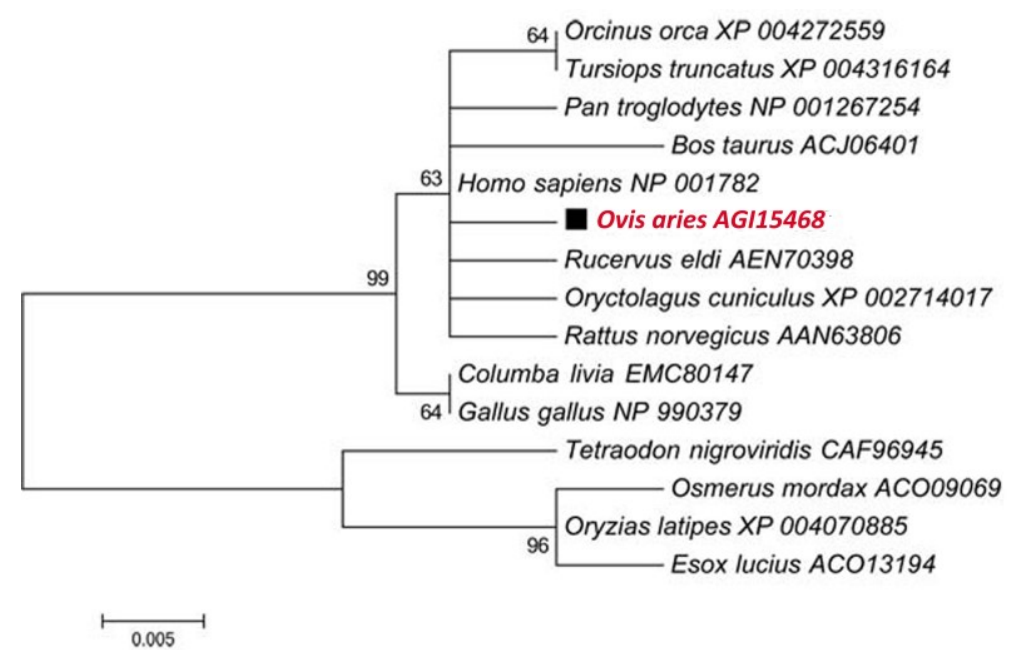

Fig. 3. Phylogenetic analysis of $\mathrm{Cdc} 42$ from sheep compared with other species. The phylogenetic tree was constructed by MAGA5.1 software using neighbour-joining (NJ) method. The numbers on the nodes reveal percentage frequencies in 1,000 bootstrap replications. The scale bar indicates 0.005 substitutions per site 
Tissue distribution of OaCde42 mRNA. RT-qPCR was performed to investigate tissue distribution of OaCdc42 mRNA in healthy tissues; $\beta$-actin was used as an endogenous control. OaCdc 42 mRNA of buffy coat as a calibrator was compared with other tissues. As a result, $\mathrm{OaCdc} 42$ mRNA was detected in all tested tissues, including buffy coat, heart, liver, spleen, lung, kidneys, rumen, small intestine, and skeletal muscles. The highest expression of OaCdc42 mRNA was detected in the intestine, followed by the liver, stomach, kidneys, heart, spleen, lung, and blood. The lowest expression was in muscles (Fig. 4).

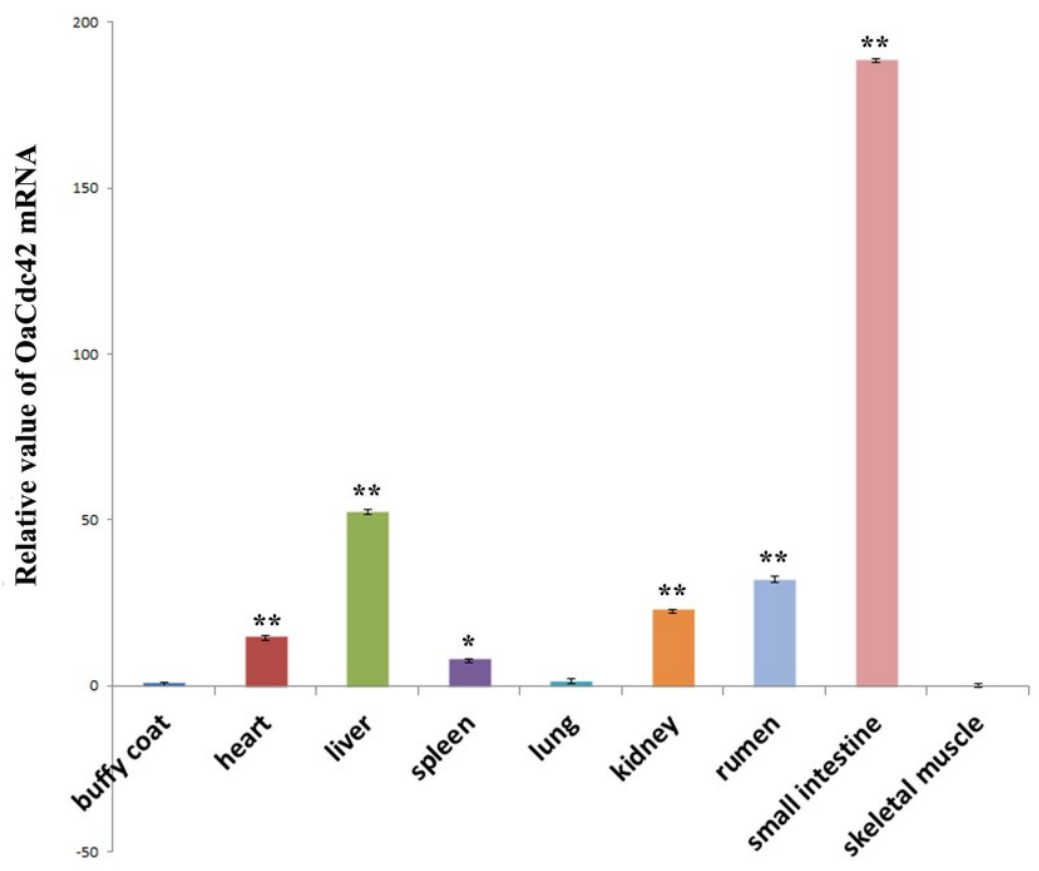

Fig. 4. Tissue distribution of OaCdc 42 mRNA from healthy sheep. The relative value of OaCdc42 mRNA was calculated using $2^{-\Delta \Delta C t}$ method and $\beta$-actin as the reference gene. Data were presented as mean $\pm \mathrm{SD}(\mathrm{n}=3, * \mathrm{P}<0.05 ; * * \mathrm{P}<0.01 v s$. buffy coat $)$. Error bars showed the SD. All tests were performed in triplicate

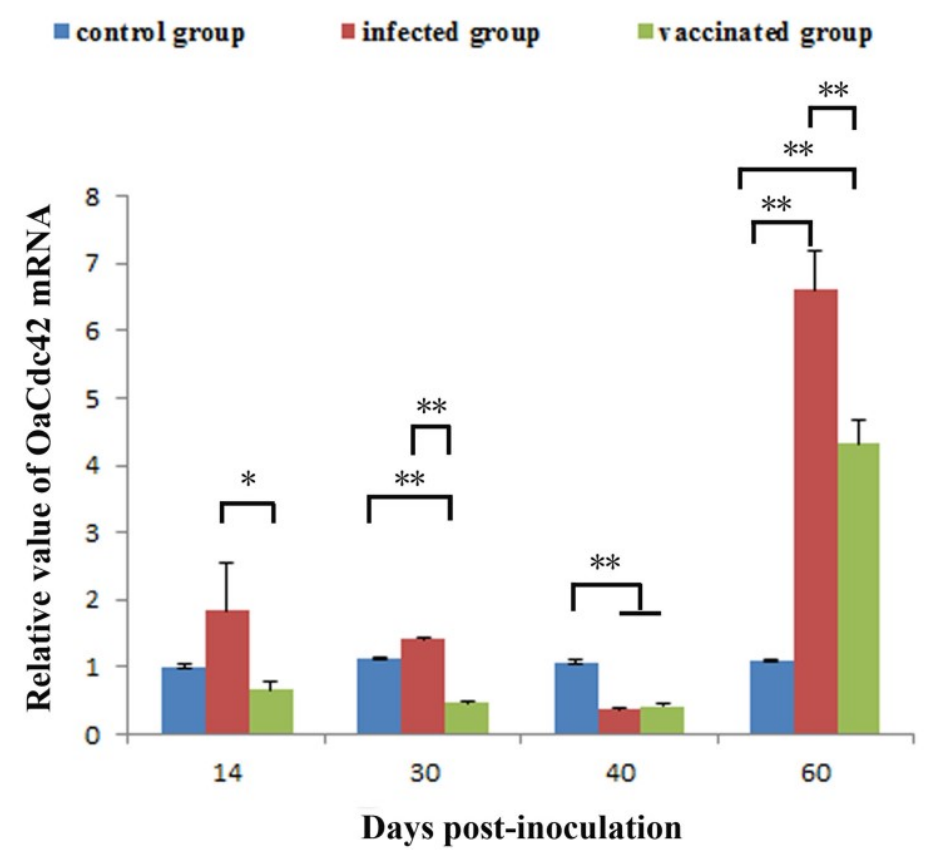

Fig. 5. The differential expression of OaCdc42 mRNA between Bm-infected sheep and S2-vaccinated sheep. Relative value of $\mathrm{OaCdc} 42 \mathrm{mRNA}$ was calculated using $2^{-\Delta \Delta \mathrm{Ct}}$ method and $\beta$-actin as the reference gene. Data were presented as mean $\pm \mathrm{SD}(\mathrm{n}=9$, $* \mathrm{P}<0.05 ; * * \mathrm{P}<0.01)$. Error bars showed the SD. All tests were performed in triplicate 
Differential expression of OaCdc42 mRNA. RT-qPCR was also carried out to investigate differential expression of $\mathrm{OaCdc} 42$ mRNA between infected and vaccinated sheep. The results showed that the expression of $\mathrm{OaCdc} 42$ mRNA differed significantly $(\mathrm{P}<0.01)$ in infected sheep or vaccinated sheep in comparison to control animals after 40 and 30 days post-inoculation, respectively. Although the level of OaCdc42 mRNA from infected sheep showed up-regulation before 30 days post-challenge, there was no significant difference compared to the control $(\mathrm{P}>0.05)$. Until 40 days post-challenge, it appeared significantly down-regulated $(\mathrm{P}<0.01)$ and up-regulated $(\mathrm{P}<0.01)$ sharply on day 60 post-challenge. OaCdc42 mRNA from vaccinated sheep was significantly down-regulated on days $30(\mathrm{P}<0.01)$ and $40(\mathrm{P}<0.01)$ post-inoculation, and up-regulated on day $60(\mathrm{P}<0.01)$ post-inoculation. However, OaCdc42 mRNA between infected group and vaccinated group was significantly different $(\mathrm{P}<0.05$ or $\mathrm{P}<0.01)$ on days 14,30 , and 60 post-inoculation and not significantly different $(\mathrm{P}>0.05)$ on day 40 post-inoculation. Moreover, the level of OaCdc42 mRNA from both infected and vaccinated group showed irregularity (Fig. 5).

\section{Discussion}

In this study, the full-length of OaCdc42 cDNA was reported and characterised for the first time. OaCdc42 cDNA was predicted to contain a conserved domain from $\mathrm{Thr}^{3}$ to $\mathrm{Leu}^{177}$, including GAP, GEF, and GDI interaction sites (Fig. 1A). GEF, GAP, and GDI were the three major regulatory proteins for regulating the cycle between the inactive GDP-bound form and active GTP-bound form, which is a typical characteristic for Rho GTPases (15). GEFs promote the exchange of GDP for GTP to activate the GTPase, whereas GAPs and GDI both negatively regulate the cycle to inactivate the GTPases. Therefore, OaCdc42 possibly has the capacity to act as a molecular switch to control signal transduction pathways by cycling between the GDP-bound form and the GTP-bound form.

The expression profile of $\mathrm{OaCdc} 42 \mathrm{mRNA}$ in healthy tissues was assessed. OaCdc42 mRNA was ubiquitously expressed in all tissue samples tested, although at a different level (Fig. 4). The broad expression profile of $\mathrm{Cdc} 42$ had been reported in buffalo (7) and swine (11). Differential expression of OaCdc42 in different tissues indicated that it may be involved in different physiological processes, such as cell growth (12), cell motility (8), apoptosis (2). The highest levels of $\mathrm{OaCdc} 42$ expressed in the intestine indicated that $\mathrm{OaCdc} 42$ must play an important role in the intestine, which needs further study.

In our study, OaCdc42 mRNA from infected or vaccinated sheep was significantly down-regulated on day 40 post-inoculation, and then significantly up- regulated 60 days post-inoculation (Fig. 5). Although OaCdc42 mRNA expression in infected sheep showed up-regulation before day 30 post-inoculation, there was no significant difference compared to the control $(\mathrm{P}>0.05)$. The results implied that $\mathrm{OaCdc} 42$ may be weakly activated by virulent $\mathrm{Bm}$, but not by avirulent $\mathrm{S} 2$. This is fully in line with the "stealthy" characteristics of Brucella. Brucella is a successful intracellular pathogen. It has evolved multiple strategies to evade immune response mechanisms to establish persistent infection and replication within hosts (1). The suppression of $\mathrm{OaCdc} 42$ mRNA may be just one of its evolved strategies. In addition, the evidence showed that $\mathrm{Cdc} 42$ was activated upon cell contact by virulent $B$. abortus, but not by non-virulent $B$. abortus. The inactivation of $\mathrm{Cdc} 42$ significantly hampered the uptake of $B$. abortus, whereas the activation of $\mathrm{Cdc} 42$ promoted the uptake of $B$. abortus (6). Thus, the activation of $\mathrm{Cdc} 42$ is required for B. abortus internalisation. However, 40 days postinoculation the level of $\mathrm{OaCdc} 42 \mathrm{mRNA}$ in infected sheep was very close to the one observed in the vaccinated sheep (Fig. 5). The reason remains elusive.

Previous studies indicated that $\mathrm{Cdc} 42$ could be activated by Salmonella through the type III secretion system (TTSS) to induce Salmonella internalisation into host cells. After its internalisation, the activation of Cdc42 is suppressed by SptP - the TTSS bacterial effector that is a GAP, namely, Salmonella reversibly activates $\mathrm{Cdc} 42$ to induce its own uptake (14). Our results showed that the expression of OaCdc 42 mRNA displayed fluctuations and oscillations (Fig. 5); the possible reason was that Brucella bacteria were able to reversibly activate $\mathrm{Cdc} 42$ to induce its own uptake.

At present, serological testing is still the primary clinical tool to diagnose brucellosis. However, lipopolysaccharide of vaccine strain of Brucella spp. is similar to the virulent strain (5), so antibodies induced by vaccine strain can interfere with serological diagnosis. Thus, routine serological diagnosis fails to distinguish vaccinated from naturally infected animals (13). It is necessary to discover a diagnostic biomarker to distinguish vaccinated animals from those naturally infected with virulent Brucella. Our results showed that the level of OaCdc42 mRNA was significantly different between infected and vaccinated sheep $(\mathrm{P}<0.05$ or $\mathrm{P}<0.01)$ on days 14,30 , and 60 postinoculation, but there was no difference $(\mathrm{P}>0.05)$ between them on day 40 post-inoculation (Fig. 5). Moreover, the expression of OaCdc42 mRNAs in infected or vaccinated sheep showed irregularity. Thus, OaCdc42 failed to distinguish vaccinated animals from the ones infected with virulent Brucella during 60 days post-inoculation. Thereby, $\mathrm{OaCdc} 42$ is not a good potential diagnostic biomarker for differential diagnosis of brucellosis.

In summary, the full-length of $\mathrm{OaCdc} 42 \mathrm{cDNA}$ was reported and characterised for the first time. OaCdc42 mRNA was widely expressed in all tested 
tissues, and the highest expression was detected in the intestine. The expression of $\mathrm{OaCdc} 42$ mRNA between vaccinated and infected sheep was significantly different $(\mathrm{P}<0.05$ or $\mathrm{P}<0.01)$ on days: 14,30 , and 60 post-inoculation, but no difference $(\mathrm{P}>0.05)$ was noted on day 40 post-inoculation. Moreover, the level of OaCdc42 mRNA from infected or vaccinated sheep showed irregularity. As our study has demonstrated, $\mathrm{OaCdc} 42$ is not a good potential diagnostic biomarker to distinguish Brucella vaccinated sheep from Brucella infected sheep.

Conflict of Interests Statement: The authors declare that there is no conflict of interests regarding the publication of this article.

Financial Disclosure Statement: This work was supported by the Natural Science Foundation of China (Grants Nos 30901070 and 31602126) and the Science \& Technology Development Project of Jilin Province, China (Grants Nos 20150204078NY and 20170101149JC).

Animal Rights Statement: The authors declare that all experiments on animals were conducted in accordance with the provisions of EU animal management practices under controllable situation (1986.11.24) and the Institutional Animal Care and Use Committee of Jilin University (SCXK2015-0004 and 20170333).

Acknowledgements: Yong-Jie Yang and Shi-Ying Lu made equal contributions to this study. Hong-Lin Ren (renhl@jlu.edu.cn) was the corresponding author.

\section{References}

1. Ahmed W., Zheng K., Liu Z.F.: Establishment of chronic infection: Brucella's Stealth Strategy. Frontiers Cell Infect Microbiol 2016, 6, 1-12.

2. Deevi R., Fatehullah A., Jagan I., Nagaraju M., Bingham V., Campbell F.C.: PTEN regulates colorectal epithelial apoptosis through Cdc42 signalling. Br J Cancer 2011, 105, 1313-1321.

3. Dorneles E.M.S., Teixeira-Carvalho A., Araujo M.S.S., Sriranganathan N., Lage A.P.: Immune response triggered by Brucella abortus following infection or vaccination. Vaccine 2015, 33, 3659-3666

4. Farhan H., Hsu V.W.: Cdc42 and cellular polarity: emercing roles at the Golgi. Trends Cell Biol 2016, 26, 241-248.

5. Franco M.P., Mulder M., Gilman R.H., Smits H.L.: Human brucellosis. Lancet Infect Dis 2007, 7, 775-786.

6. Guzman-Verri C., Chaves-Olarte E., von Eichel-Streiber C., Lopez-Goni I., Thelestam M., Arvidson S., Gorvel J.P., Moreno E.: GTPases of the Rho subfamily are required for Brucella abortus internalization in nonprofessional phagocytes - direct activation of Cdc42. J Biol Chem 2001, 276, 4443544443.

7. Hao Y., Zhang X., Zhang D., Cheng Y., Du L., Kuang W., Lei M., Jiao H., Qi C., Wang F.: Molecular cloning and tissue expression of cell division cycle 42 in buffalo tissue. J Anim Vet Adv 2012, 11, 1018-1022.

8. Hu J.H., Mukhopadhyay A., Craig A.W.B.: Transducer of Cdc42-dependent actin assembly promotes epidermal growth factor-induced cell motility and invasiveness. J Biol Chem 2011, 286, 2261-2272.

9. Keestra A.M., Winter M.G., Auburger J.J., Fraessle S.P., Xavier M.N., Winter S.E., Kim A., Poon V., Ravesloot M.M., Waldenmaier J.F.T., Tsolis R.M., Eigenheer R.A., Bauemler A.J.: Manipulation of small Rho GTPases is a pathogen-induced process detected by NOD1. Nature 2013, 496, 233-237.

10. Lee K., Boyd K.L., Parekh D.V., Kehl-Fie T.E., Baldwin H.S., Brakebusch C., Skaar E.P., Boothby M., Zent R.: Cdc42 promotes host defenses against fatal infection. Infect Immun 2013, 81, 2714-2723.

11. Liu G.Y., Gao S.Z., Ge C.R., Zhang X.: cDNA cloning and tissue expression analyses of the encoding regions for three novel porcine genes-MJD1, CDC42, and NECD. Anim Biotechnol 2008, 19, 117-121.

12. Melendez J., Liu M., Sampson L., Akunuru S., Han X., Vallance J., Witte D., Shroyer N., Zheng Y.: Cdc42 coordinates proliferation, polarity, migration, and differentiation of small intestinal epithelial cells in mice. Gastroenterology 2013, 145, 808-819.

13. Nielsen K., Smith P., Widdison J., Gall D., Kelly L., Kelly W., Nicoletti P.: Serological relationship between cattle exposed to Brucella abortus, Yersinia enterocolitica O:9, and Escherichia coli O157:H7. Vet Microbiol 2004, 100, 25-30.

14. Patel J.C., Galan J.E.: Differential activation and function of Rho GTPases during Salmonella-host cell interactions. J Cell Biol 2006, 175, 453-463.

15. Raftopoulou M., Hall A.: Cell migration: Rho GTPases lead the way. Dev Biol 2004, 265, 23-32.

16. Rigano L.A., Dowd G.C., Wang Y., Ireton K.: Listeria monocytogenes antagonizes the human GTPase $\mathrm{Cdc} 42$ to promote bacterial spread. Cell Microbiol 2014, 16, 1068-1079.

17. Swaine T., Dittmar M.T.: CDC42 use in viral cell entry processes by RNA viruses. Viruses-Basel 2015, 7, 6526-6536.

18. Wang J.-Y., Wu N., Liu W.-H., Ren J.-J., Tang P., Qiu Y.-H., Wang C.-Y., Chang C.-D., Liu H.-J.: A repA-based ELISA for discriminating cattle vaccinated with Brucella suis 2 from those naturally infected with Brucella abortus and Brucella melitensis. Mol Cell Probes 2014, 28, 251-254.

19. Watson L.J., Rossi G., Brennwald P.: Quantitative analysis of membrane trafficking in regulation of $\mathrm{Cdc} 42$ polarity. Traffic 2014, 15, 1330-1343.

20. Xu J.-D., Jiang H.-S., Wei T.-D., Zhang K.-Y., Wang X.-W., Zhao X.-F., Wang J.-X.: Interaction of the small GTPase Cdc42 with arginine kinase restricts white spot syndrome virus in shrimp. J Virol 2017, 91, e01916-16.

21. Xu Y., Qi Y., Luo J., Yang J., Xie Q., Deng C., Su N., Wei W., Shi D., Xu F., Li X., Xu P.: Hepatitis B virus X protein stimulates proliferation, wound closure and inhibits apoptosis of HuH-7 cells via CDC42. Int J Mol Sci 2017, 18, 586-599.

22. Yang Y.J., Liu Z.S., Lu S.Y., Li C., Hu P., Li Y.S., Liu N.N., Tang F., Xu Y.M., Zhang J.H., Li Z.H., Feng X.L., Zhou Y., Ren H.L.: Molecular cloning, expression and characterization of programmed cell death 10 from sheep (Ovis aries). Gene 2015, $558,65-74$. 